\title{
EOP's: ¿ Cuánto reducen la función pulmonar los beta bloqueantes en pacientes con enfermedad pulmonar obstructiva crónica?
}

\section{Escenario clínico}

Un paciente de 75 años tabaquista y portador de enfermedad pulmonar obstructiva crónica (EPOC) sufre un infarto agudo de miocardio de cara anterior. Al alta de Unidad Coronaria queda medicado con aspirina, atenolol, enalapril y atorvastatina. Recibe además bromuro de ipratropio y agonistas beta 2 por vía inhalatoria para el EPOC. Al poco tiempo, su disnea aumenta de clase funcional I a clase funcional II. El médico que lo atiende se plantea varios diagnósticos diferenciales. Piensa que una de las posibilidades es que los betabloqueantes hayan disminuido el el estado funcional respiratorio y ésta sea la causa de la progresión de los síntomas.

\section{Pregunta que generó el caso}

En los pacientes con EPOC (población), la administración de beta bloqueantes (intervención) disminuye la función pulmonar y/o aumentan la disnea (resultado)

\section{Estrategia de búsqueda}

Se realizó una búsqueda en MEDLINE empleando como palabras clave " Chronic Obstructive Pulmonary disease AND beta blocker" y limitando a revisiones sistemáticas o meta-análisis de menos de 5 años desde su publicación.

Se hallaron dos citas, de las cuales se seleccionó la que parecía apropiada para responder la pregunta.

\section{EPOC y beta bloqueantes}

La Guía de la American Herat Association y del American College of Cardiology para prevención secundaria del infarto agudo de miocardio recomienda iniciar beta bloqueantes en todos los pacientes post-infarto y continuarlos indefinidamente ${ }^{1}$ bloqueantes en el período post-infarto, menos de la mitad de los pacientes los reciben crónicamente ${ }^{2}$. La resistencia de los médicos para prescribirlos se relaciona con la percepción de menor eficacia de la droga en la era de los antiagregantes, los inhibidores de la enzima convertidora de angiotensina (IECA), las estatinas y los procedimientos de revascularización; la preocupación acerca de la seguridad en pacientes con insuficiencia cardíaca, EPOC diabetes mellitus o edad avanzada y una percepción de menor eficacia en los infartos sin elevación del segmento $\mathrm{ST}^{3}$.

Varios ensayos clínicos han mostrado que los beta bloqueantes reducen la mortalidad en los pacientes infartados. El mayor estudio fue el BHAT 4 que demostró que el propranolol redujo en un $26 \%$ la mortalidad global comparado con placebo. El Norwegian Multicenter Study Group mostró que el timolol redujo un 39\% la mortalidad y un $28 \%$ el riego de reinfarto ${ }^{5}$.

Estos estudios son previos a la introducción de varias drogas que hoy son de uso rutinario y excluyeron pacientes con insuficiencia. Con el empleo de IECA, varios médicos cuestionaron el empleo cardíaco de beta bloqueantes en los pacientes con infarto agudo de miocardio. Sin embargo dos grandes ensayos ${ }^{6,7}$ mostraron que los beta bloqueantes confieren protección adicional más allá de la que proporcionan los IECA en forma aislada. Un meta-análisis

de 31 estudios encontró que los beta bloqueantes tienen beneficios aun en los pacientes que reciben fibrinolíticos y aspirina. Más aun, los beneficios de los beta bloqueantes son mayores que otras terapias. El número de pacientes necesarios para tratar (NNT) para prevenir una muerte en un período de 2 años es de 24 con fibrinolíticos y aspirina, de 42 con beta bloqueantes, 94 con estatinas y 153 con antiagregantes ${ }^{8}$.

Ya ha sido comentado que la presencia de contraindicaciones es un motivo para no prescribir beta bloqueantes en estos pacientes. Una revisión de 200.000 historias clínicas en el Cooperative Cardiovascular Project encontró que sólo el 34\% de los pacientes recibían beta bloqueantes luego del infarto agudo de miocardio ${ }^{9}$. El porcentaje era aun menor en ancianos, y en pacientes con bradicardia, baja presión arterial, diabetes tipo 1, insuficiencia cardíaca y EPOC. Incluso en estos subgrupos, la mortalidad era menor si se administraban beta bloqueantes ${ }^{10}$.

La preocupación acerca de que los beta bloqueantes puedan causar broncoconstricción hace que los médicos no indiquen o lo hagan en dosis subóptimas a los pacientes infartados con EPOC o asma.

En el caso que nos interesa existe la preocupación sobre la posibilidad de que los beta bloqueantes sean los causantes del empeoramiento de los síntomas del paciente.

\section{Resumen de la Evidencia}

Fuente: Salpeter S, Ormiston T, Salpeter E, et al. Cardioselective beta-blockers for chronic obstructive pulmonary disease. Cochrane Database Syst Rev. 2002; (2): CD003566 (latest version 18 octubre 2001)

\section{Objetivo}

Evaluar el efecto de los betabloqueantes selectivos en la función pulmonar de los pacientes con EPOC. Evaluar si los beta bloqueantes cardioselectivos afectan la respuesta a los beta 2 agonistas.

\section{Estrategia de búsqueda}

Se buscaron los ensayos clínicos aleatorizados, controlados y ciegos publicados desde 1966 hasta mayo de 2001. Las bases consultadas fueron EMBASE/Excerpta Medica, MEDLINE y CINAHL. También se buscaron resúmenes de simposios y referencias de los estudios identificados.

\section{Selección de estudios para ser incluidos en el meta-análisis}

Los estudios debían ser aleatorizados, controlados y ciegos. Debían evaluar el efecto de beta bloqueantes cardioselectivos por vía oral o intravenosa en la función pulmonar medida por el volumen espiratorio forzado en un segundo (VEF1). EI VEF1 podía estar expresado como litros o como porcentaje del teórico ideal. Otra manera en que la función pulmonar podía estar evaluada era a través de los síntomas en pacientes con EPOC (definidos como pacientes con una obstrucción fija, con menos de $80 \%$ de VEF 
teórico medido de manera basal).

Dos investigadores independientes evaluaron el diseño de los estudios, extrajeron los datos acerca de las características de los pacientes, intervención, grupo control y resultados (cambios en el VEF1, respuesta a los beta agonistas suministrados luego de indicar atenolol o placebo y el reporte de los pacientes sobre síntomas (sibilancias, tos o disnea). Para el análisis se usaron solo datos publicados.

\section{Resultados principales}

Diecinueve ensayos cumplían los criterios de inclusión $(n=267)$, de estos, 17 ( $n=226$ ) incluyeron un grupo control con placebo. Se reportaron sólo los resultados de estos 17 estudios. Los beta bloqueantes empleados fueron atenolol, metoprolol, bisoprolol, practolol, celiprolol y acebutolol.

Los beta bloqueantes cardioselectivos no produjeron cambios significativos en el VEF1 comparados con placebo administrados como dosis única (Diferencia Media Ponderada [DMP] ${ }^{*}-2.05 \%$ de VEF1 [IC95\% -6.05 a $1.96 \%$ ]) o como tratamiento de mayor duración (DMP -2.55\% ìC95\% -5.94 a 0.84\%]).

Tampoco se hallaron diferencias en los síntomas antre beta bloqueantes y placebo. En estudios de corto plazo, un meta-análisis de 9 ensayos $(n=114)$ no encontró diferencias en los síntomas (diferencia de riesgo* 0.0 [IC95\% -0.03 a 0.03$]$ ). En ensayos de mayor duración un meta-análisis de 7 ensayos $(n=98)$ no mostró diferencias en los síntomas (diferencia de riesgo* 0.0 , IC 95\% 0.04 a 0.04 ).

Tampoco se encontraron diferencias de VEF1 a corto o a largo plazo en pacientes que venían recibiendo agonistas beta 2 inhalatorios.

\section{Conclusión de los autores}

En ensayos que enrolaron en total menos de 300 pacientes, los beta bloqueantes cardioselectivos no redujeron la función pulmonar medida por VEF1 en pacientes con EPOC ni afectaron la respuesta a los agonistas beta 2 inhalatorios. Dado su beneficio demostrado en condiciones como insuficiencia cardíaca, arritmias e hipertensión, los mismos no deben dejar de administrarse en pacientes con enfermedad pulmonar reactiva leve a moderada.

\section{Comentario y conclusión para la práctica}

Este estudio refuerza el siguiente mensaje: los beta bloqueantes no están contraindicados en los pacientes con EPOC. Esta conclusión es importante si tenemos en cuenta que un $20 \%$ de los pacientes infartados tienen EPOC o asma ${ }^{10}$ y muchos de los pacientes con EPOC tienen a su vez enfermedad cardíaca isquémica. Ya ha sido comentado que en estos pacientes los beta bloqueantes salvan vidas.

Este meta-análisis muestra la pobre evidencia que hay en el tema: pocos ensayos, pocos pacientes, muchos ensayos sin placebo y en general con un seguimiento corto.

Los resultados de este meta-análisis son concordantes con la información observacional acerca de que el empleo de beta bloqueantes no aumenta las hospitalizaciones por reagudización de EPOC ${ }^{11}$. También son similares a un meta-análisis de los mismos autores publicado recientemente ${ }^{12}$. En el mismo, para evaluar el efecto específico en pacientes con EPOC, se analizaron diez ensayos que incluyeron solo pacientes con esta patología. No se encontraron diferencias significativas en el VEF1 en ensayos de dosis única $(-5.28 \%$ [IC95\%, $-10.03 \%$ a $-0.54 \%])$ o con tratamiento continuo $(1.07 \%$ [IC $95 \%,-3.3 \%$ a $5.44 \%])$, y no hubo aumento de síntomas en ningún ensayo.

Sin embargo no existe evidencia suficiente del efecto de los beta bloqueantes en pacientes con EPOC a largo plazo.

La sugerencia es monitorear de cerca de los pacientes con EPOC a los que se indican beta bloqueantes. Se sugiere comenzar con bajas dosis y titularla; y emplear beta bloqueantes cardioselectivos de vida media corta como el atenolol o metoprolol.

A pesar de que los estudios no han mostrado que estas drogas deterioran la función pulmonar o exacerban los síntomas, debido al bajo número de pacientes y al corto seguimiento de los estudios, es prudente considerar que los beta bloqueantes puedan ser la causa de la exacerbación de síntomas en pacientes con EPOC en ausencia de una explicación alternativa. En ese caso, el tratamiento debe ser reevaluado tomando en cuenta los daños y beneficios.

\section{Dra. Karin Kopitowski [ Unidad de Medicina Familiar y Preventiva. Hospital Italiano de Buenos Aires ]}

\footnotetext{
Referencias

1. Behar S, Panosh A, Reicher-Reiss $\mathrm{H}$, et al. Prevalence and prognosis of chronic obstructive pulmonary disease among 5839 consecutive patients with acute myocardial infarction with chronic obstructive pulmonary disease or asthma. Am J Med. 1992; 93: 637-641

2. Smith SC Jr, Blair SN, Bonow RO, et al. AHA/ACC Scientific Statement: AHA/ACC guidelines for preventing heart attack and death in patients with atherosclerotic cardiovascular disease: 2001 update. A statement for healthcare professionals from the American Heart Association and the American College of Cardiology. Circulation. $2001 ; 104: 1577-1579$. 3. Krumholz HM, Radford MJ, Wang Y, et al. National use and effectiveness of beta-blockers for the treatment of elderly patients after acute myocardial infarction: National Cooperative Cardiovascular Project. JAMA. 1998; 280: 623-629.

4. Bradford WD, Chen J, Krumholz HM. Under-utilisation of beta-blockers after acute myocardial infarction: pharmacoeconomic implications. Pharmacoeconomics. 1999; 15: 257-268. 5. Beta-Blocker Heart Attack Trial Research Group. A randomized trial of propranolol in patients with acute myocardial infarction: II. Morbidity results. JAMA. 1983; 250: $2814-2819$. 6. Norwegian Multicenter Study Group. Timolol-induced reduction in mortality and reinfarction in patients surviving acute myocardial infarction. N Engl J Med. $1981 ; 304: 801-807$. 7. Vantrimpont P, Rouleau JL, Wun CC, et al. Additive beneficial effects of beta-blockers to angiotensin-converting enzyme inhibitors in the Survival and Ventricular Enlargement (SAVE) Study. SAVE Investigators. J Am Coll Cardiol. 1997; 29: 229-236.

8. Spargias KS, Hall AS, Greenwood DC, et al. Beta-blocker treatment and other prognostic variables in patients with clinical evidence of heart failure after acute myocardial infarction: evidence from the AIRE study. Heart. 1999; 81: 25-32.

9. Freemantle N, Cleland J, Young P, et al. Beta-blockade after myocardial infarction: systematic review and meta-regression analysis. BMJ. 1999; 318: 1730-1737.

10. Gottlieb SS, McCarter RJ, Vogel RA. Effect of beta-blockade on mortality among high-risk and low-risk patients after myocardial infarction. N Engl J Med. 1998; 339: 489-497.

11. Chen J, Radford MJ, Wang Y, et al. Effectiveness of beta-blocker therapy after acute myocardial infarction in elderly patients with chronic obstructive pulmonary disease or asthma. J Am Coll Cardiol. 2001; 37: 1950-1956.

12. Salpeter S, Ormiston T, Salpeter E, et al. Cardioselective beta-blockers in patients with reactive airway disease: a meta-analysis. Ann Intern Med. 2002 Nov 5;137(9):715-25.
} 